\title{
RO Membrane to Remove Sulfate: an Inland Brackish Water Desalination Pilot Study
}

\author{
Qigang Chang ${ }^{1, *}$, Brian R. Bergantine ${ }^{1}$, Robert (Bo) Johnson ${ }^{2}$, Srinivas (Vasu) \\ Veerapaneni ${ }^{2}$, Troy B. Hall ${ }^{3}$, Mark A. Peterson ${ }^{3}$ and David Buchholz ${ }^{3}$ \\ ${ }^{1}$ Advanced Engineering and Environmental Services, Inc. (AE2S), USA \\ ${ }^{2}$ Black \& Veatch, USA \\ ${ }^{3}$ City of Fargo, Water Treatment Plant, Fargo, ND, USA
}

\begin{abstract}
The City of Fargo completed a Facility Plan of their Water Treatment Plant (WTP) in 2011 to address two main issues: increasing water demands and high sulfate concentrations within a raw water source (Sheyenne River) primarily due to Devils Lake flooding. Reverse Osmosis (RO) was recognized as the most appropriate technology for sulfate reduction, and recommended for use in the WTP expansion. An RO pilot study was performed to evaluate its feasibility for two operational scenarios. RO membranes experienced rapid fouling in the Polishing Scenario, which used RO to further treat filtered water from the existing WTP (pretreatment, lime softening, ozone, and granular filtration). RO membranes exhibited superior performance in the Parallel Scenario, which was a separate treatment process (coagulation/flocculation/sedimentation + microfiltration/ultrafiltration $+R O$ ) parallel to the existing WTP. RO membrane autopsies indicated that membrane fouling was organic and biological for the Polishing Scenario while organic and scaling for the Parallel Scenario. Optimization studies were performed in the Parallel Scenario to determine optimal coagulation conditions for pretreatment as well as flux, recovery, and membrane cleaning regimes for both the MF/UF and the RO. Uniquely, an RO membrane selection pilot was conducted for both scenarios to evaluate RO membranes from four different manufacturers. The slight difference surface chemistry among various RO membrane can cause substantial different performance. It was found that one RO membrane could not be cleaned adequately, although it has many successful applications elsewhere. This one year pilot study proved that RO technology is feasible to reduce sulfate concentrations to acceptable levels in the City's finished water.
\end{abstract}

Keywords: RO membrane, sulfate, fouling, surface water, microfiltration, ultrafiltration.

\section{INTRODUCTION}

The Devils Lake basin was created by the last advance of the continental ice sheets in North Dakota (ND). As one of the largest natural water bodies in ND, Devils Lake has been continuously rising from a water surface elevation of 439.7 meter in 1993 to 443.3 meter on June 27, 2011, as shown in Figure 1. Devils Lake flooding has destroyed hundreds of homes and businesses and inundated thousands of acres of productive farmland. To alleviate the substantial social and economic impact, the State of ND has constructed emergency outlets to discharge Devils Lake water into the Sheyenne River. The west-end emergency outlet was built in 2005 and expanded to $7.1 \mathrm{~m}^{3} / \mathrm{s}$ in 2010 . An east-end emergency outlet with a capacity of $9.9 \mathrm{~m}^{3} / \mathrm{s}$ was operational in 2012, for a combined outlet capacity of $17 \mathrm{~m}^{3} / \mathrm{s}$. In addition, an emergency control structure has been constructed to control a catastrophic overflow from the lake into the Sheyenne River.

Operation of the emergency outlets has helped alleviate flooding in the closed basin; however,

*Address correspondence to this author at the Advanced Engineering and Environmental Services, Inc. (AE2S); Tel: +1 218-299-5610; Fax: +1 218-2995611; E-mail: qigang.chang@ae2s.com, qgchang@gmail.com problems related to water quality have also resulted from the emergency outlets. Devils Lake has high concentrations of dissolved solids (TDS) currently ranging from $1,100 \mathrm{mg} / \mathrm{L}$ up to $2,600 \mathrm{mg} / \mathrm{L}$. The elevated TDS concentration is because the lake is a terminal lake, meaning that water will only leave the lake through evaporation, plant uptake, or ground infiltration, and only when the lake elevation is high enough will water overflow into the Sheyenne River. Release of the high TDS water (including high sulfate), causes a degradation of the downstream water quality in the Sheyenne River, which is used as a drinking water source for the City of Fargo (City), the largest city in ND. A United States Geological Survey study predicted that the sulfate concentration in the Sheyenne River could reach $750 \mathrm{mg} / \mathrm{L}$ in the lower Sheyenne River at Fargo with the two emergency outlets operating at $17 \mathrm{~m}^{3} / \mathrm{s}$ [1]. The historic average sulfate concentration in the Sheyenne River at Fargo is approximately $160 \mathrm{mg} / \mathrm{L}$, and the secondary standard in drinking water (Secondary Maximum Contaminant Level (SMCL)) is $250 \mathrm{mg} / \mathrm{L}$ [2]. This anticipated elevated sulfate concentration causes concerns to municipal water supply systems that rely on the Sheyenne River as major water source, such as the City. 


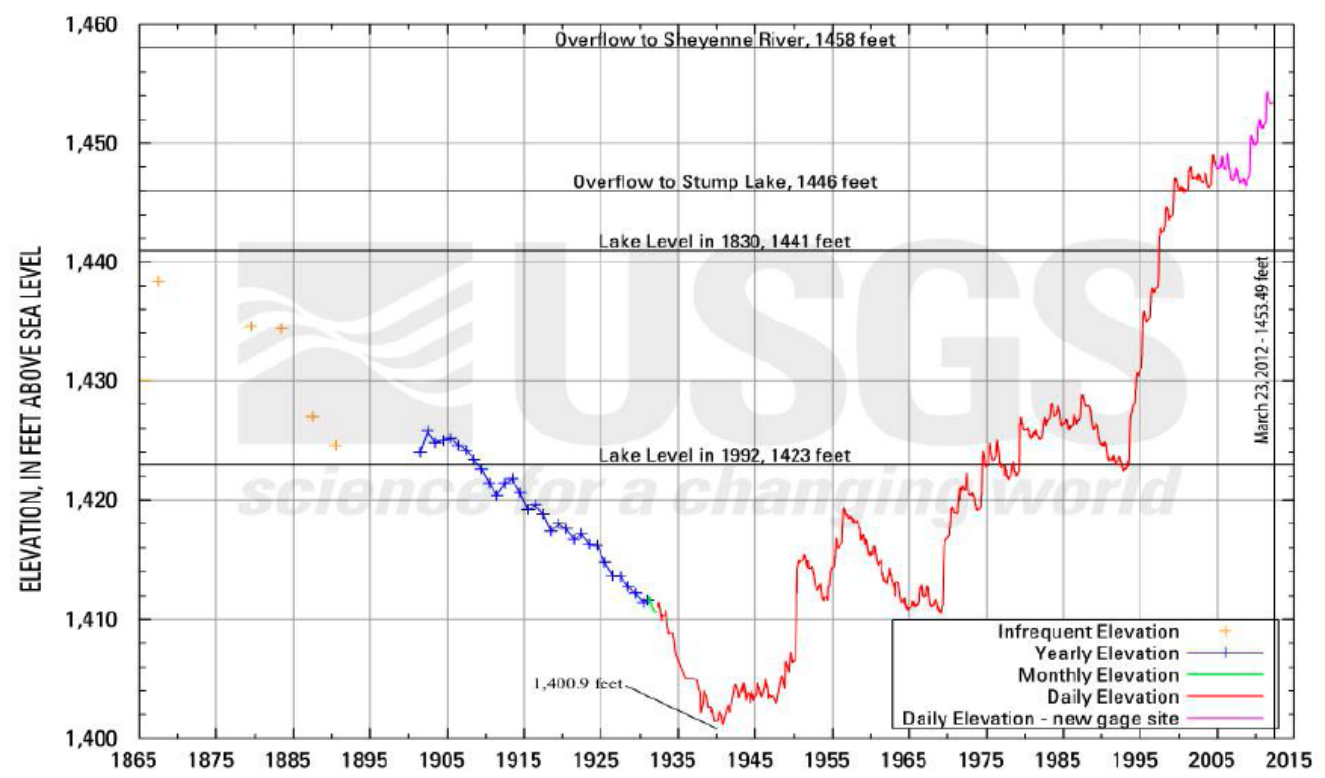

Figure 1: The Devils Lake Historical Water Level (1 ft= 0.305 meter).

The City currently operates a lime-softening surface water treatment plant (WTP) constructed in 1997 with a rated capacity of $113,562 \mathrm{~m}^{3} /$ day. The City obtains its raw water from both the Red River and Sheyenne River, and strategically selects the proper sources or a blend of sources to provide its customers with highquality drinking water and minimize treatment costs. From 2006 to 2010, the WTP has relied on the Sheyenne River as its water resource approximately $40 \%$ of the time. This dramatic water quality change in the Sheyenne River is a concern to the City because its existing WTP is unable to remove sulfate. The City actively sought solutions to address the anticipated water quality change to meet its commitment to providing safe and high-quality drinking water to its customers. As such, the City retained Advanced Engineering and Environmental Services, Inc. (AE2S), in association with Black \& Veatch (B\&V), to complete a Facility Plan to evaluate potential improvements to the WTP.

In the Facility Plan [3], several treatment technologies were evaluated to remove sulfate and the reverse osmosis (RO) technology was recommended as the most appropriate alternative for sulfate removal. However, the performance of various RO membranes can vary, particularly with respect to fouling, the unique characteristics of the water being treated, and membrane chemistry. In addition optimization of pretreatment requirements and RO system design parameters, such as optimal permeate flux and recovery, is desired prior to design of a full-scale project. To address these issues, the City initiated a
Pilot Study in July 2011. Due to dramatic seasonal variations in water quality in the source water experienced in ND, the pilot study has been conducted for a year to investigate both cold and warm seasonal impacts, as well as to catch the seasonal characteristics of the Sheyenne River water quality, particularly the spring runoff.

\section{OBJECTIVES AND METHODOLOGY}

The objectives of the Pilot Study were to evaluate the performance of RO membranes under two scenarios, evaluate various pretreatment configurations, and determine the optimal design and operating parameters to meet treated water quality goals. Specifically, the objectives of the Pilot Study included:

- $\quad$ Evaluate the performance and the effectiveness of the proposed treatment processes in meeting the targeted finished water quality goals.

- Establish full-scale design and operating parameters for the proposed processes.

- Determine the pretreatment requirements for downstream processes through optimization of chemical, dose, $\mathrm{pH}$, etc.

- $\quad$ Determine the potential for membrane fouling and means of mitigating fouling.

- $\quad$ Project the anticipated cleaning frequencies and long-term performance of the membrane processes. 
- Evaluate relative performance of various membrane elements with respect to rejection and fouling potential.

\section{Pilot Study Setup}

Six improvement alternatives were evaluated in the Facility Plan for the City of Fargo WTP [3]. It was determined that two testing scenarios could effectively pilot test all of the improvement alternatives that utilized RO. The two testing scenarios were: (1) the Polishing Pilot Scenario (designed to test a new RO membrane polishing plant to further treat filtered water from the existing WTP); and (2) the Parallel Pilot Scenario (designed to test a new RO membrane to treat raw water directly and blend with the existing WTP). The Pilot Study was designed to evaluate the effectiveness of both the pilot scenarios (Figure 2). Additionally, an RO membrane selection (ROMS) pilot was developed for both scenarios to examine the performance of various RO membranes from different manufactures. In total, four unique pilot tests were investigated as part of the Pilot Study, and included the Polishing Pilot, the Polishing ROMS Pilot, the Parallel Pilot, and the Parallel ROMS Pilot.

The Polishing Pilot Scenario was designed to evaluate performance of the RO process when treating filtered, but not disinfected, water from the existing WTP. The treated water was piped from the filter effluent and was a variable mixture of water from both the Red and Sheyenne Rivers.

The Parallel Pilot Scenario tested a complete treatment process that would operate in parallel to the existing WTP. The treatment processes that were piloted consisted of pretreatment, microfiltration/ ultrafiltration (MF/UF), and RO. The Parallel Pilot Scenario had three additional objectives: (1) determine the optimal operating conditions of the coagulation process, plate settler, and MF/UF system; (2) evaluate the suitability of the pretreated water as RO feed water (fouling potential); and (3) optimize the RO operating flux and maximize recovery.

One unique aspect of this Pilot Study is that equipment used for both of the ROMS pilots was designed for both testing scenarios. At first, the Polishing ROMS Pilot was fed with the same water as the Polishing Pilot, and later the Parallel ROMS Pilot was fed with same water as the Parallel RO system that was pretreated through coagulation, sedimentation, and MF/UF filtration.

The existing WTP has a chemical lab that is certified to perform most water quality analysis required by the Pilot Study. This was a huge advantage because samples could by analyzed quickly; allowing engineers and WTP staff to optimize operations of the Pilot Study accordingly.
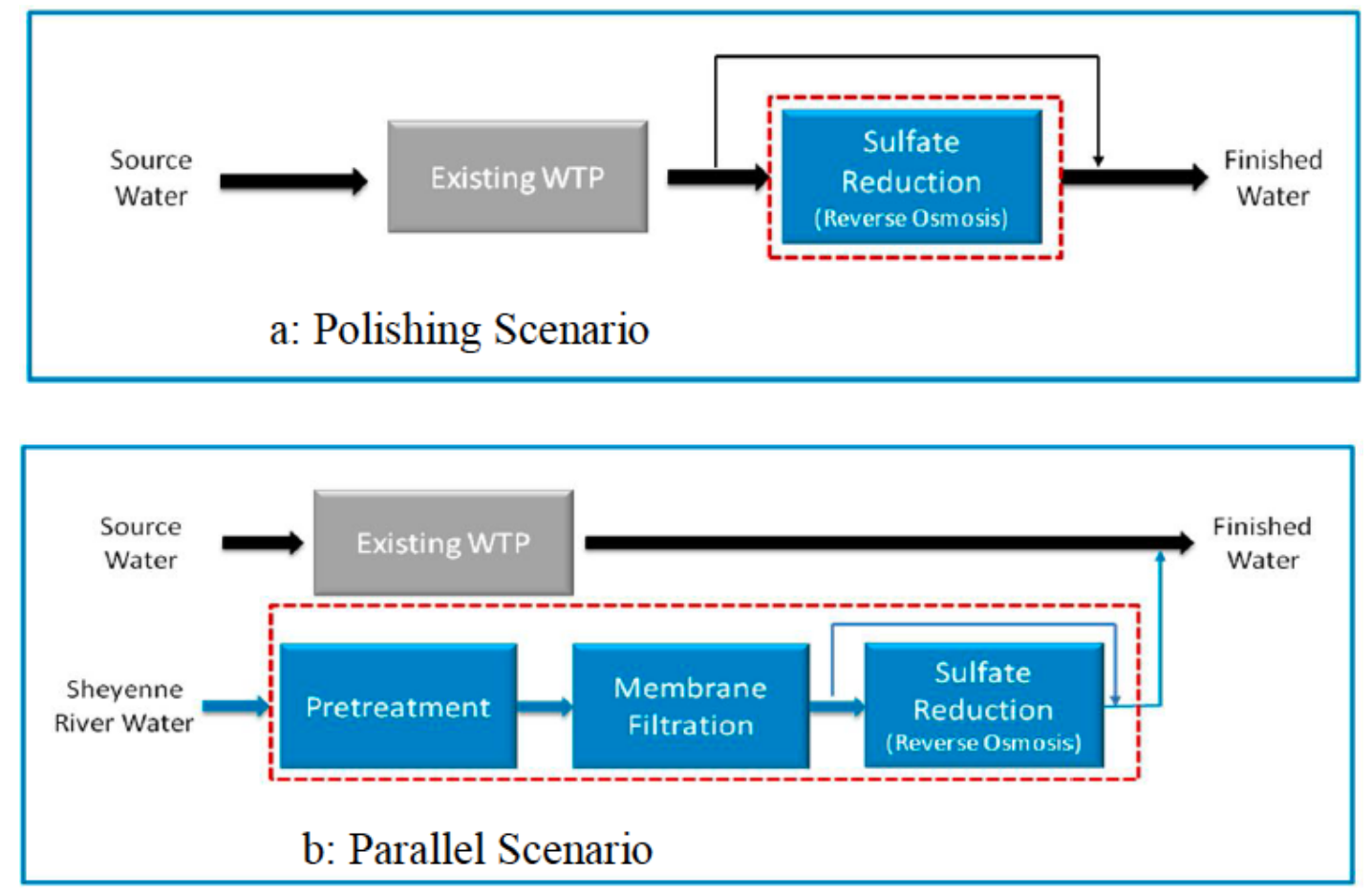

Figure 2: Fargo WTP Pilot Study Scenarios. 
Table 1: MF/UF Membrane Characteristics

\begin{tabular}{|c|c|}
\hline Properties/Specifications & Values \\
\hline \hline Material & PVDF \\
\hline Membrane Area & $38 \mathrm{~m}^{2}$ \\
\hline Nominal Pore Size & $0.02 \mu \mathrm{m}$ \\
\hline Surface Properties & Non-ionic and hydrophilic \\
\hline Flow Path & Outside-in \\
\hline Transmembrane Pressure (TMP) Range & $<=0.9$ bar \\
\hline Operating pH Range & $5.0-9.0$ \\
\hline Max. Operating Temperature & $40{ }^{\circ} \mathrm{C}$ \\
\hline Cleaning pH Range & $2.0-11.0$ \\
\hline Max. Chlorine Concentration & $1,000 \mathrm{mg} / \mathrm{L}$ \\
\hline
\end{tabular}

\section{Pilot Study Equipment}

Equipment used in this Pilot Study included two 2stage RO Pilot skids (identical skids for both Polishing and Parallel Pilots), a ROMS skid (for both Polishing and Parallel ROMS Pilots), a MF/UF skid (for the Parallel Pilot), and a pretreatment pilot skid (for the Parallel Pilot). Each type of equipment used in the Pilot Study is described in following sections.

\section{Pretreatment Unit}

The pretreatment unit, provided by Meurer Research Inc (MRI) of Golden, CO, was a selfcontained plate settler unit complete with inline mixer, rapid mix, 3-stage flocculator, inlet diffuser, plate settler, and sludge removal. The parallel scenario pretreatment unit was rated for $25 \mathrm{~m}^{3} / \mathrm{h}$, but the weir tubes were capped to provide an equivalent loading rate of $0.7 \mathrm{~m} / \mathrm{h}$ for the inclined plate settler at the desired flow of $17 \mathrm{~m}^{3} / \mathrm{h}$. Settled solids collection was performed by a MRI Hoseless Cable-Vac collector.

\section{MF/UF Skid}

GE Zenon ZeeWeed 1000 membrane was piloted in the Parallel Pilot Scenario. The MF/UF skid consisted of a main control unit, a membrane tank (3-stack), a strainer $(400 \mu \mathrm{m})$, and a clean-in-place (CIP) system. Table 1 summarizes the properties and specifications of the ZeeWeed 1000 membrane.

\section{RO Skids}

\section{2-Stage RO Skids}

The skids contained two stages of pressure vessels in a 2:2:1:1 array and each vessel contained three 4inch diameter by 40 -inch long RO elements. A $5 \mu \mathrm{m}$ cartridge filter was provided to remove any large particles that may damage the RO membranes. The feed water was fed into a high pressure pump on variable frequency drive (VFD) to boost the pressures for the RO elements.

\section{ROMS Skid}

The ROMS skid was divided into two trains and each train contained two pressure vessels. The skid provides flexibility to investigate four different $\mathrm{RO}$ membranes under various antiscalants and clean-inplace regimes. The ROMS skid was controlled through a manual control panel with pump control switches and a series of manual needle valves, flow meters, pressure gauges, and $\mathrm{pH} /$ temperature probes.

\section{RESULTS AND DISCUSSIONS}

\section{Source Water Quality}

During the Pilot Study, an intensive source water quality monitoring program was conducted. Samples taken from different locations were analyzed at desired frequency (from weekly to monthly) to evaluate the performance of each individual unit. Table 2 summarizes the average water quality in the one year piloting period. The raw water to the parallel scenario was straight Sheyenne River water while the polishing train treated water from the full-scale plant and it was a blending of the Red and Sheyenne River. The Sheyenne River source water quality presents a challenge to RO membrane technology with high concentrations of total organic carbon (TOC), TDS, and wide range of temperature $\left(1.7-27^{\circ} \mathrm{C}\right)$.

\section{Polishing ROMS Pilot}

Four different RO membranes (Dow, GE, Hydranautics, and Toray) were tested. The rapid membrane fouling was observed on all four RO 
Table 2: Average Source Water Quality

\begin{tabular}{|c|c|c|c|c|c|}
\hline \multirow{2}{*}{$\begin{array}{c}\text { Water Quality } \\
\text { Parameter }\end{array}$} & \multirow[t]{2}{*}{ Units } & \multicolumn{2}{|c|}{ Polishing Scenario } & \multicolumn{2}{|c|}{ Parallel Scenario } \\
\hline & & Raw & RO Feed & Raw & RO Feed \\
\hline Al & $\mathrm{mg} / \mathrm{L}$ & 0.43 & 0.05 & 0.48 & 0.01 \\
\hline $\mathrm{Ba}$ & $\mathrm{mg} / \mathrm{L}$ & $\mathrm{n} / \mathrm{a}$ & 0.03 & $\mathrm{n} / \mathrm{a}$ & 0.05 \\
\hline $\mathrm{Ca}$ & $\mathrm{mg} / \mathrm{L}$ & 71.9 & 44.8 & $\mathrm{n} / \mathrm{a}$ & 96.7 \\
\hline $\mathrm{Cl}$ & $\mathrm{mg} / \mathrm{L}$ & $\mathrm{n} / \mathrm{a}$ & 24.4 & $\mathrm{n} / \mathrm{a}$ & 60.3 \\
\hline $\mathrm{Fe}$ & $\mathrm{mg} / \mathrm{L}$ & 0.52 & 0.03 & 0.29 & 0.06 \\
\hline $\mathrm{Mg}$ & $\mathrm{mg} / \mathrm{L}$ & 42.1 & 12.5 & $\mathrm{n} / \mathrm{a}$ & 53.7 \\
\hline $\mathrm{Mn}$ & $\mathrm{mg} / \mathrm{L}$ & 0.17 & 0.02 & 0.21 & 0.20 \\
\hline $\mathrm{pH}$ & & 8.4 & 8.8 & 8.2 & $7.2-7.5$ \\
\hline $\mathrm{PO}_{4}^{-3}$ & $\mathrm{mg} / \mathrm{L}$ & 0.86 & 1.00 & 0.50 & 0.28 \\
\hline $\mathrm{SiO}_{2}$ dissolved & $\mathrm{mg} / \mathrm{L}$ & 6.6 & 6.8 & 16.8 & 15.7 \\
\hline $\mathrm{SiO}_{2}$ total & $\mathrm{mg} / \mathrm{L}$ & 6.6 & 6.8 & 22.0 & 16.0 \\
\hline $\mathrm{SO}_{4}^{-2}$ & $\mathrm{mg} / \mathrm{L}$ & 188 & 220 & 334 & 336 \\
\hline TOC & $\mathrm{mg} / \mathrm{L}$ & 8.48 & 5.00 & 8.96 & 6.57 \\
\hline Total Alkalinity & $\mathrm{mg} / \mathrm{L}$ & 103 & 104 & 358. & 301 \\
\hline TDS & $\mathrm{mg} / \mathrm{L}$ & $\mathrm{n} / \mathrm{a}$ & 486 & $\mathrm{n} / \mathrm{a}$ & 879 \\
\hline
\end{tabular}

membranes. As shown in Figure 3, one of the RO membranes could not be adequately cleaned with either the caustic or acidic CIPs with a moderate cleaning regime $\left(30{ }^{\circ} \mathrm{C}\right.$ at $\mathrm{pH} 3$ or 11$)$. However, the other three RO membranes were well cleaned following this protocol. An aggressive cleaning protocol $\left(40{ }^{\circ} \mathrm{C}\right.$ at $\mathrm{pH} 2$ or 12) was tested and it was found that the poorly cleaned RO membrane still could not be cleaned. This RO membrane is a popular RO membrane that has been successful in many other surface water applications. This observation confirms that the performance of an RO membrane system is site specific and a pilot study is essential for successful membrane applications.

The salt passage of one of the four RO membranes was found to increase slightly after each CIP. At the end of five-month Polishing ROMS Pilot, the salt passage of this RO membrane reached an unacceptable level of $5 \%$. The membrane autopsy indicated that the structure of this RO membrane was destroyed, which is most likely due to the frequent aggressive CIPs.

\section{Polishing Pilot}

The Polishing Pilot 2-stage RO skid was installed with one of four RO membranes being piloted in the Polishing ROMS Pilot and the initial operational parameters included a membrane flux of 14 gallon per square foot per day (gfd) at a recovery of $85 \%$. It was found that the normalized permeate flow dropped more than $15 \%$ over a period of 14 to 28 days, which is shorter than 90 days anticipated in the pilot protocol. According to the bio-film observed on the cartridge filters, bio fouling was identified as one of the major RO fouling mechanisms. A recent study reported that the assimilable organic carbon (AOC) was $1.9 \mathrm{mg} / \mathrm{L}$ in the ozone contact basin effluent and $0.58 \mathrm{mg} / \mathrm{L}$ in the filtered water at the Fargo WTP [4]. High AOC encourage the bio-growth, especially in the warmer summer months and can well explain the quick biofouling observed in Polishing RO pilot. Various biofouling mitigation measures, including a low concentration of chloramines in the RO feed water, were tested, but unfortunately the rapid RO membrane fouling could not be alleviated.

The rapid RO membrane fouling forced investigators to reduce permeate flow to lower flux rate and increase RO concentrate flow to lower recovery. In addition to the initial flux of $14 \mathrm{gfd}$ at $85 \%$ recovery, flux $12 \mathrm{gfd}$ at $85 \%$ recovery and $11 \mathrm{gfd}$ at $75 \%$ recovery were tested as well. Unfortunately, the lower flux rates and lower recovery did not improve the RO performance as expected. At the end of the Polishing Pilot, an RO membrane autopsy was performed and it was found that the majority of the RO membrane fouling was caused by organic fouling. The ozonation 


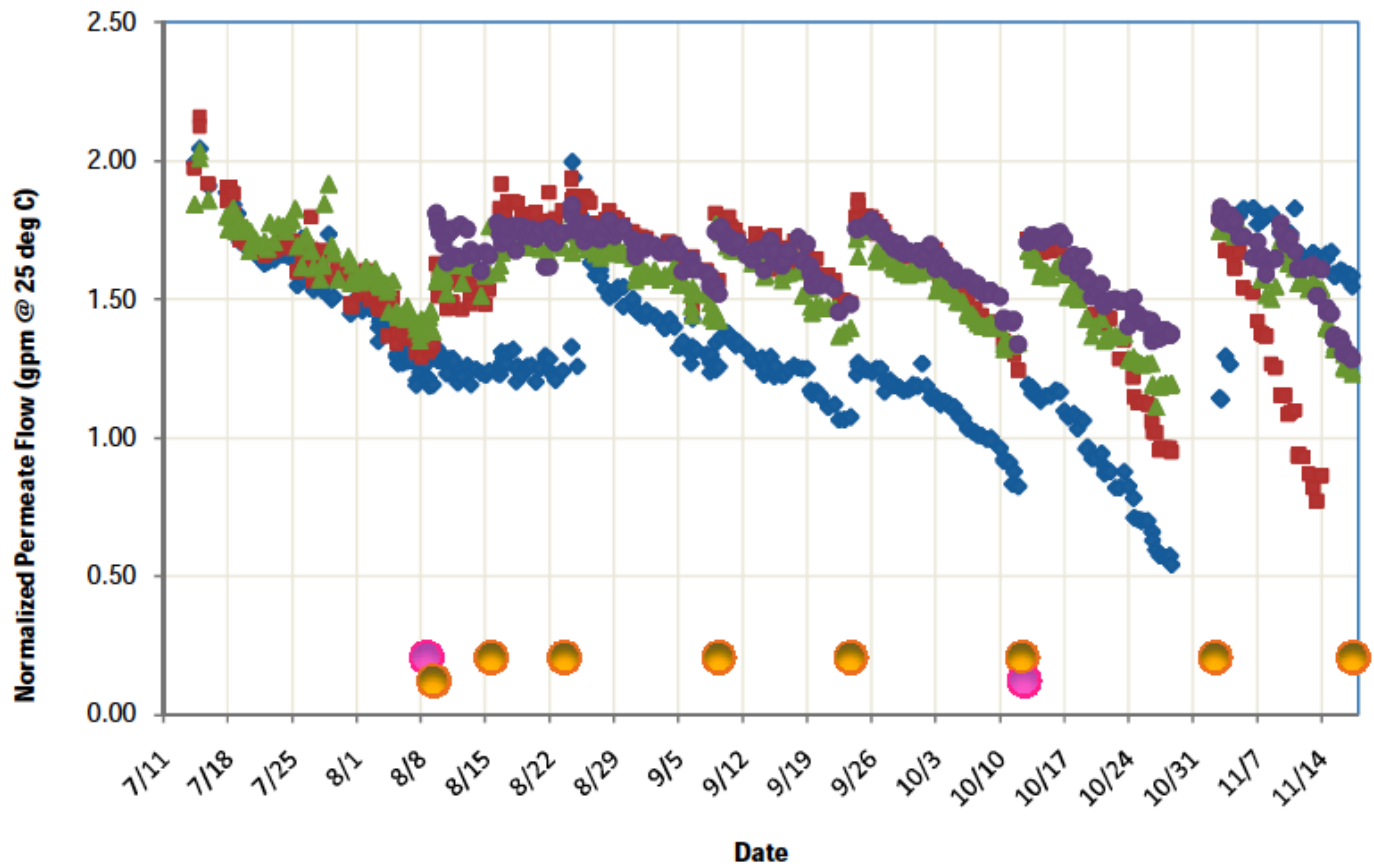

$\diamond$ PV1 Norm Perm Flow $=$ PV2 Norm Perm Flow $\Delta$ PV3 Norm Perm Flow • PV4 Norm Perm Flow Acidic CIP Caustic CIP

Figure 3: Polishing ROMS (1 gpm=227 L/h).

process of the WTP not only increased the portion of AOC, but also changed the fraction of TOC. A previous study investigated the natural organics fractionation and found that the portion of hydrophilic neutral increased significantly after the ozonation process [5]. The hydrophilic natural portion of organics was reported to cause the greatest $\mathrm{RO}$ membrane fouling [6].

In addition to the TOC analysis, silt density index (SDI) tests were performed daily to evaluate the fouling potential of the $\mathrm{RO}$ feed water. The $\mathrm{SDl}_{15}$ ranged between four and five during the piloting period, which also indicates a high fouling tendency due to colloidal particles.

The rapid RO membrane fouling resulted in frequent caustic clean-in-places (CIPs) at a rate of approximately every four to six weeks. Replacement of cartridge filters also occurred every four to six weeks. This could ultimately lead to higher chemical and operation and maintenance (O\&M) costs as well as decreased membrane life.

\section{Parallel Pilot}

The Parallel Pilot consisted of three sub-pilot components: the Pretreatment Pilot Unit, the MF/UF Pilot Unit, and the RO Parallel Pilot Unit. During the one year pilot period, two coagulants were tested in the Pretreatment Pilot Unit. Coagulant dose and coagulation $\mathrm{pH}$ were optimized with regards to turbidity and TOC reduction. Five different instantaneous fluxes were tested on the MF/UF Pilot Unit to determine the optimal flux for full-scale implementation. Different CIP regimes and operational parameters (flux and recovery) were evaluated on the 2-stage RO Parallel Pilot Unit.

\section{Pretreatment Pilot Unit}

The treatment goals of the Pretreatment Pilot Unit were to maintain a clarified water turbidity of less than 3 NTU $95 \%$ of the time, and to provide a TOC removal of $25-30 \%$. During the Pilot Study, the Pretreatment Pilot Unit was continually operated at a hydraulic loading rate of $0.7 \mathrm{~m} / \mathrm{h}$. Coagulant trials were conducted for both ferric chloride and polyaluminum chloride (PACl) in an effort to maintain the Pretreatment Pilot Unit goals. The performance of the Pretreatment Pilot Unit was optimized through a series of laboratory jar tests and in-situ tests. Figure 4 presents the optimization of coagulation $\mathrm{pH}$ and coagulant dose of $\mathrm{PACl}$ with regarding to the TOC reduction. The figure indicates that different combinations of $\mathrm{pH}$ and dose can reach the same level of TOC reduction. This information along with the costs of the coagulant and acid (for $\mathrm{pH}$ adjustment) will 


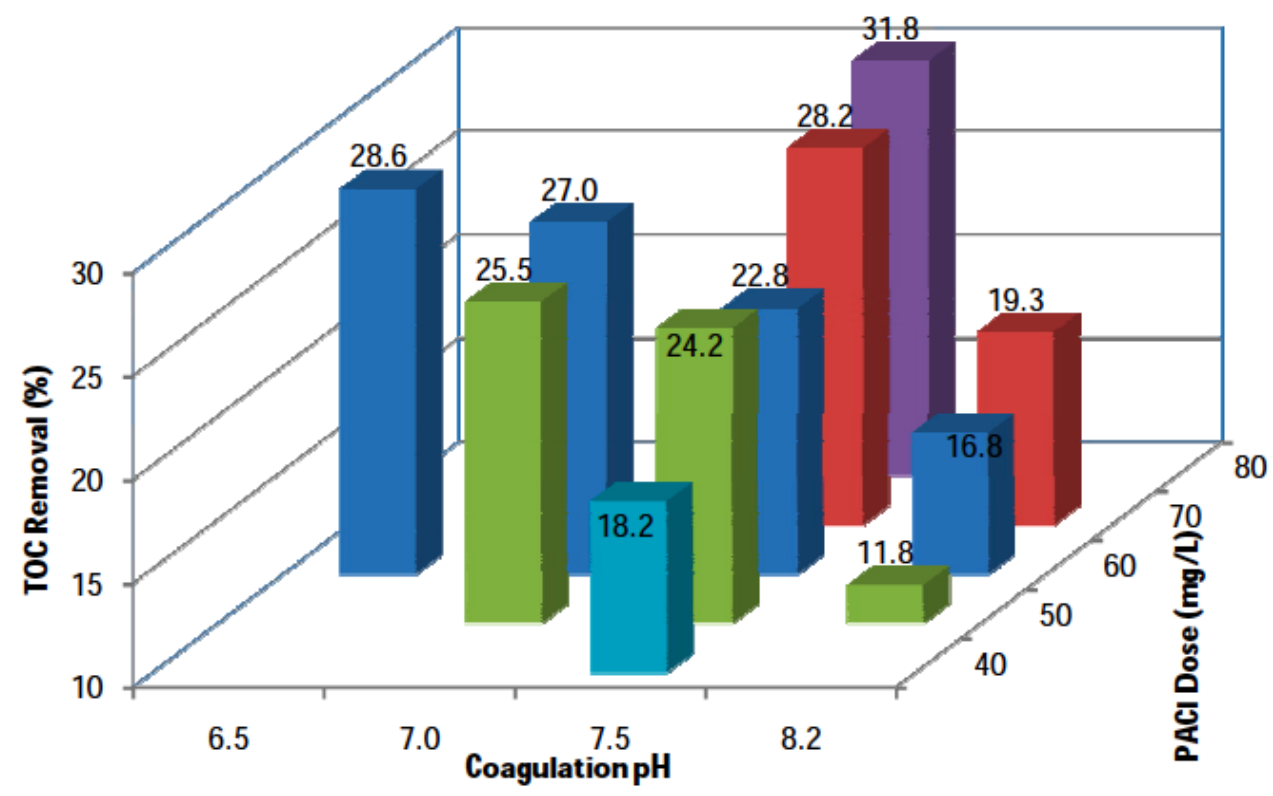

Figure 4: Pretreatment Pilot Unit Optimization with PACl.

enable the future full-scale membrane WTP to select the most cost-effective manner to operate the pretreatment unit. The ferric chloride solution used for the Pretreatment Pilot Unit was a strong acid solution that could suppress $\mathrm{pH}$ down to 7.5 without the addition of acid. However, the ferric chloride solution also contained a significant enough concentration of manganese to be problematic to downstream membrane equipment.

\section{MF/UF Pilot Unit}

The MF/UF Pilot Unit was operated at different instantaneous flux ranging from 25 to $40 \mathrm{gfd}$ through testing period. This flux range is equivalent to $23-55 \mathrm{gfd}$ when corrected to a temperature of $20{ }^{\circ} \mathrm{C}$. The performance of the MF/UF Pilot Unit was evaluated on several parameters including: recovery, filtrate water quality (turbidity and SDI), permeability, membrane fouling, cleaning (maintenance wash interval, CIP interval, and CIP efficiency), and fiber breakage.

The MF/UF Pilot Unit recovery was maintained at $95 \%$ with the exception of a short duration where the MF/UF Pilot Unit backwash waste was recycled back through the Pretreatment Pilot Unit, which effectively increased the recovery above 95\%. No significant impacts were noticed during the recycle of the MF/UF backwash waste and a full-scale system should be able to achieve a recovery greater than $95 \%$. The MF/UF Pilot Unit filtrate turbidity was consistently below 0.03 NTU and the $\mathrm{SDI}_{15}$ averaged 2.62 over the length of the pilot testing based on 66 measurements.
During the one year piloting period, MF/UF membrane fouling had not been an issue. The temperature corrected transmembrane pressure (TMP) progressively increased from 0.21 to 0.48 bar during most of the piloting cycles (usually six weeks). During the cold winter months, the highest TMP was 0.69 bar at instantaneous flux of $40 \mathrm{gfd}$, which was still within the recommended TMP operation range of less than 0.9 bar. The backwash interval was 38 to 40 minutes with a backwash duration of less than 2 minutes. The backwashes generally reduced the TMP by 0.03 to 0.06 bar. The chemical enhanced maintenance washes were twice a week for most of the piloting period and was increased to four times per week for a short period. However, no improvements were observed by the increased frequency of chemical enhanced maintenance washes. The performance of the MF/UF Pilot Unit are presented in Figure $\mathbf{5}$.

The CIP protocol of $2000 \mathrm{mg} / \mathrm{L}$ citric acid solution at $\mathrm{pH} 2$ and $600 \mathrm{mg} / \mathrm{L}$ sodium hypochlorite was employed and the CIP interval was able to be maintained at approximately 42 days. As shown in Figure 6, both citric acid and sodium hypochlorite contributed to membrane permeability recovery, which implies that the membrane fouling was a combination of organic and inorganic. Overall, the CIP protocol employed in the pilot study can recover $80-90 \%$ of the initial membrane permeability. Membrane integrity testing (MIT) was performed daily on the MF/UF Pilot Unit and none of these MIB test failed. No fiber breaks occurred during the one year piloting period. According to 


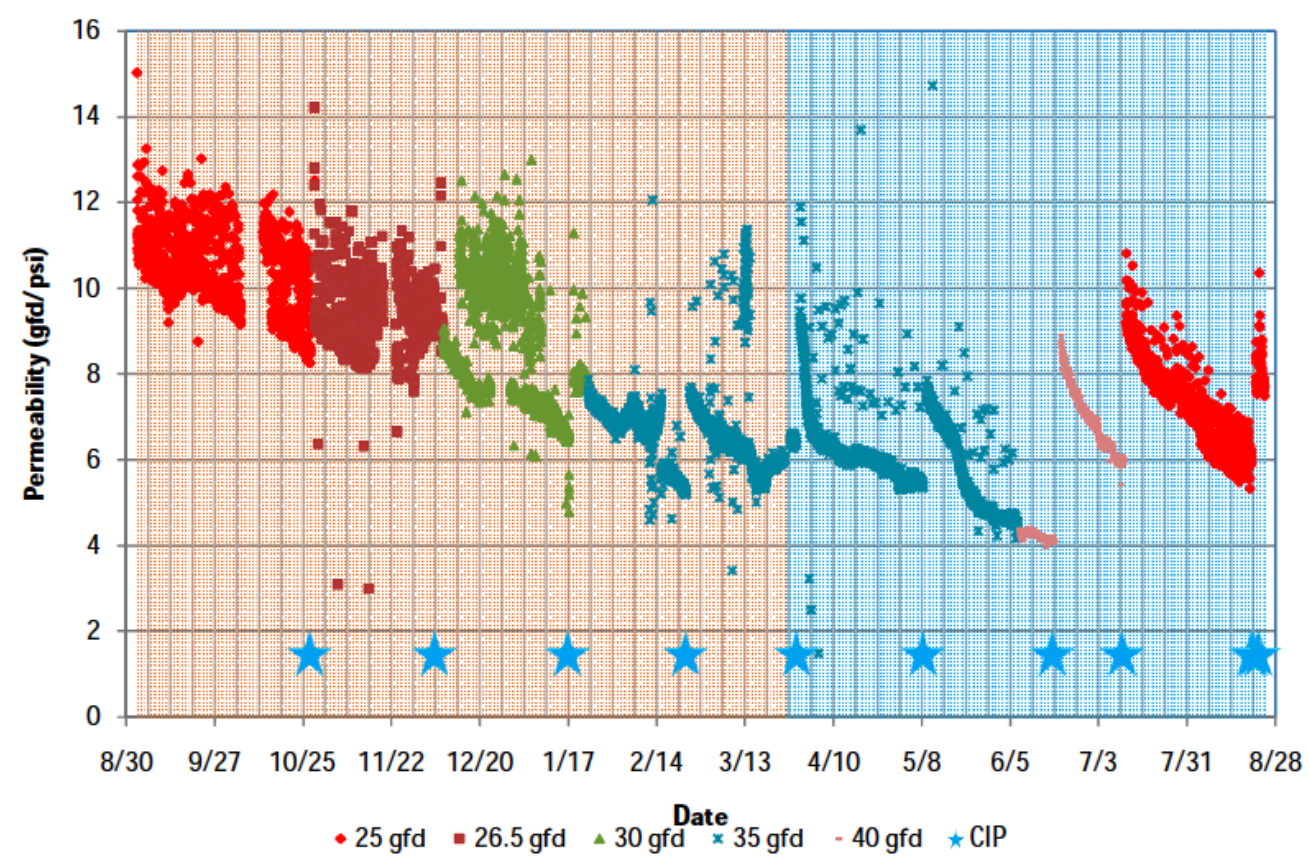

Figure 5: MF/UF Pilot Unit Temperature Corrected Permeability.

(orange indicates the period using ferric chloride as coagulant in the pretreatment unit while the blue indicates PACl).

Zeeweed 1000 membrane California Challenge test and previous project, this membrane is expected to be awarded minimum of 4 Log removal for Cryptosporidium, 4 Log Removal for Giardia, and 0.5 Log inactivation for virus.

\section{RO Parallel Pilot Unit}

The Parallel RO Pilot Unit was installed with the same RO membrane as the Polishing Pilot in order to evaluate two scenarios in a comparable fashion. With experiences from previous projects, the flux was set at $14 \mathrm{gfd}$ at $85 \%$ recovery initially. A modulating valve was installed in the first stage permeate line of this RO pilot skid enabling engineers to operate two stages at the same flux as well as to analyze performance of the two stages separately. As shown in Figure 7, the parallel RO unit was performing well for the first seven months (September to April). The RO membrane

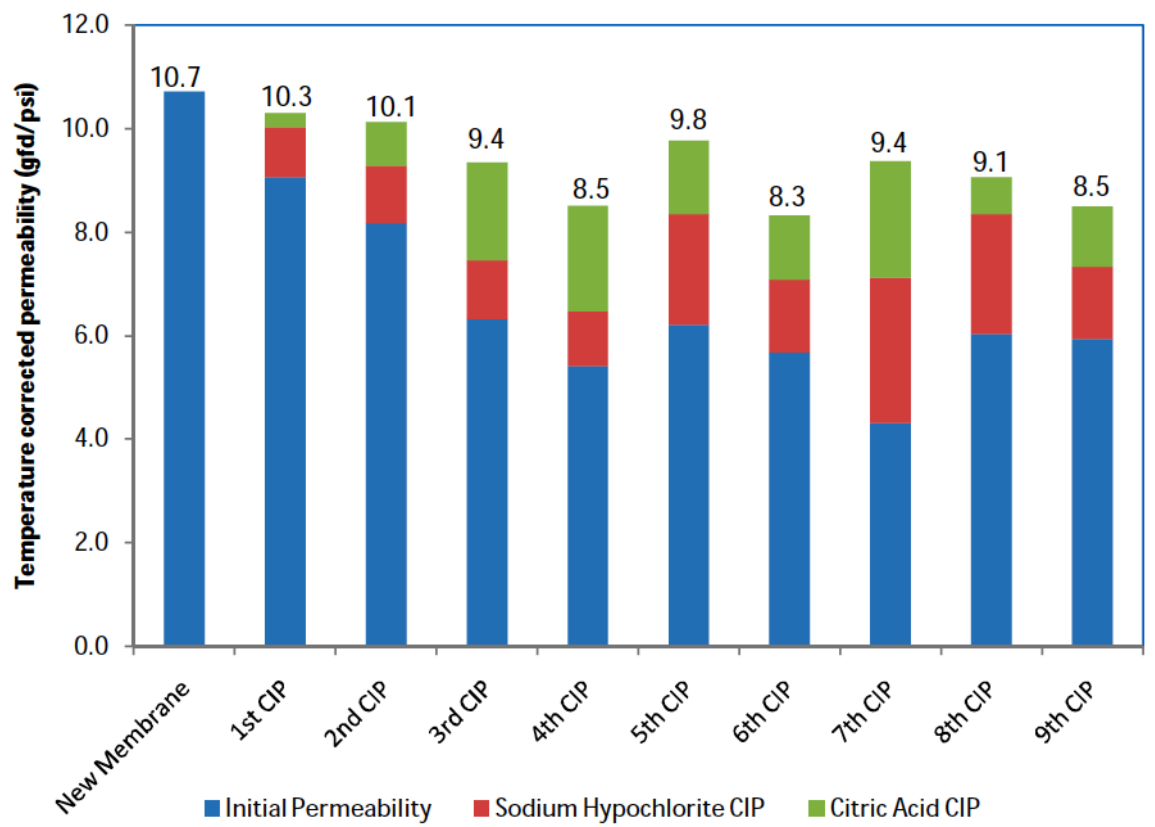

Figure 6: MF/UF CIP Permeability Recovery. 


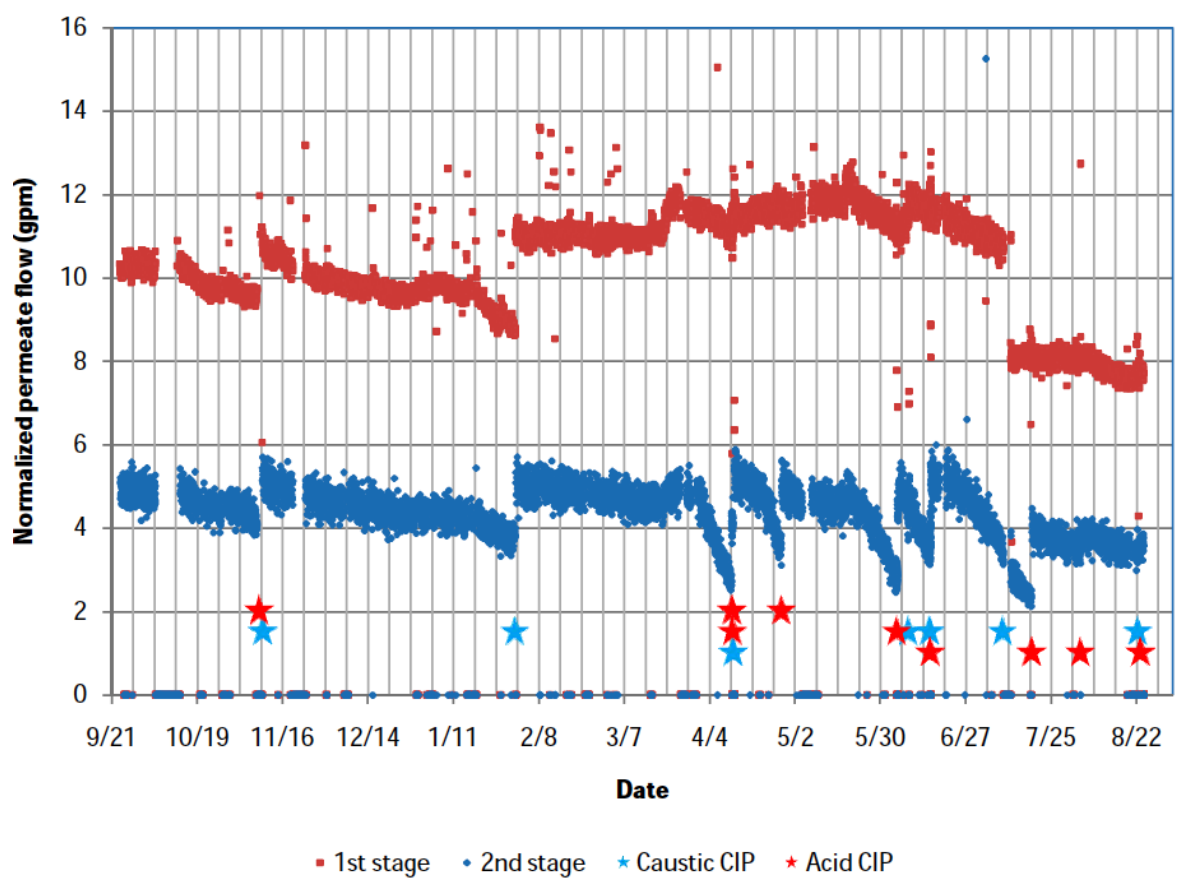

Figure 7: Parallel Pilot RO Performance $(1 \mathrm{gpm}=227.1 \mathrm{~L} / \mathrm{h})$.

experienced a slow progressive membrane fouling resulting in CIP frequency approximately every 10 to 12 weeks for acid CIP and 12 to 16 weeks and caustic CIP. As shown in Figure 7, both acid and caustic CIPs were performed in early November even though there was a minimal drop of normalized permeate flow. This CIP was performed because the antiscalant feed pump air-locked over a weekend and the differential pressure jumped approximately 0.41 bar. At this time, the investigators elected to clean the RO membranes to mitigate the conversion of inorganic fouling to irreversible fouling.

During the spring and summer with snowmelt runoff and rainfall events, RO membrane in the second stage experienced relatively quick fouling. During this period, the water quality data showed that the Sheyenne River water quality changed rapidly. The raw water total hardness jumped from $300 \mathrm{mg} / \mathrm{L}$ to $500 \mathrm{mg} / \mathrm{L}$ over a weekend. The rapidly changing water quality presented a challenge to correctly adjust the antiscalant dose. To prevent the RO membrane from being permanently fouled, the CIPs were performed once the normalized permeate flow dropped more than $15 \%$. Because of this criterion, these CIPs were primarily performed on the second stage. As shown in Figure 7, the RO membrane can be well cleaned through acid CIPs while caustic CIP benefit was marginal. This implies that the fouling in the second stage is largely due to scaling. This was confirmed by the RO membrane autopsy at the end of the Pilot Study. Figure 8 indicates that some silica particles with size of $1-10 \mu \mathrm{m}$ were identified on the RO membrane surface by the Energy Dispersive X-Ray (EDX) analysis. In the last month of the Parallel Pilot, the recovery was reduced from $85 \%$ to $82 \%$ and it helped to mitigate the scaling in the second stage. Sulfuric acid was fed prior to RO skid to suppress $\mathrm{pH}$ and it was found that low $\mathrm{pH}$ also helped to alleviate the scaling in the second stage.

\section{Parallel ROMS Pilot}

With the knowledge from the Polishing ROMS pilot, three membrane manufacturers provided the same RO membrane modules for Parallel ROMS pilot while one membrane vendor recommended testing a different $R O$ membrane that was designed with high fouling resistance. The Parallel ROMS Pilot lasted 11 weeks and all four membranes performed better in the Parallel ROMS Pilot as compared to the Polishing ROMS Pilot as shown in Figure 9. This is likely due to the consistent water quality from the pretreatment processes (clarified water from the Pretreatment Pilot Unit and treatment with the MF/UF Pilot Unit).

An interesting observation is that the same RO membrane that cannot be cleaned in the Polishing ROMS Pilot also experienced cleaning difficulties in the Parallel ROMS Pilot. During the Parallel ROMS Pilot, one RO membrane was running at a feed pressure of 0.69 bar higher than other three probably due to the tightness of the RO membrane elements. The salt 


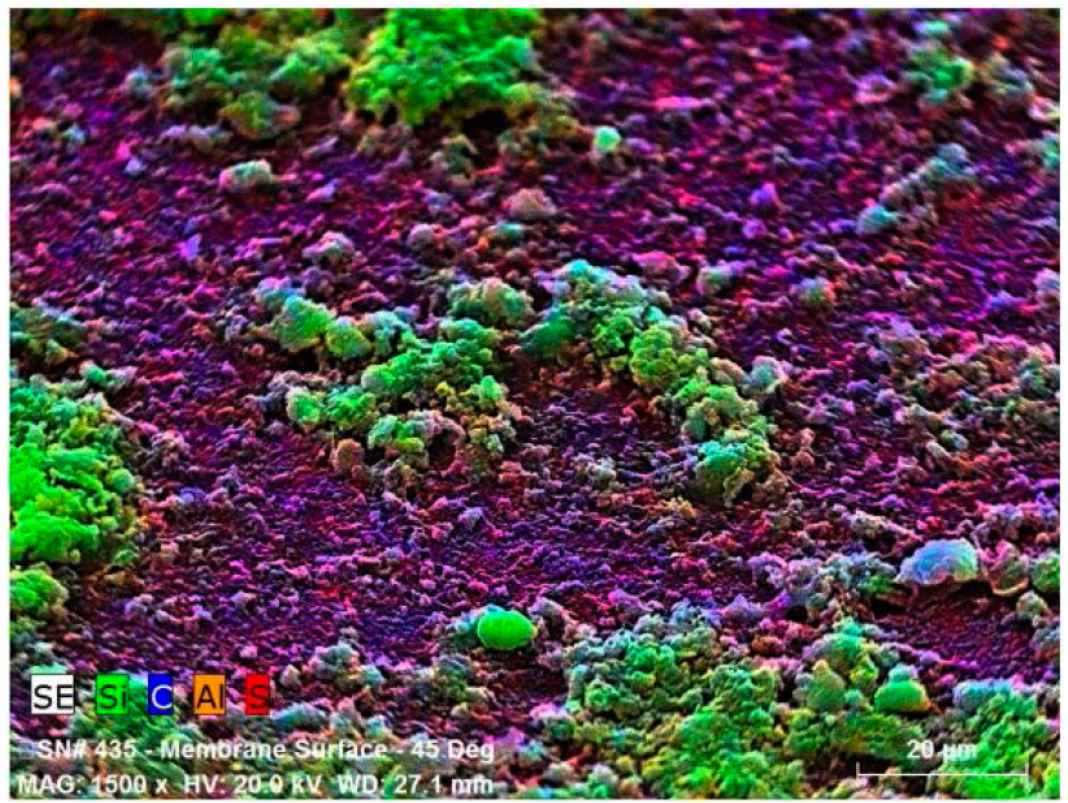

Figure 8: RO Membrane Surface Chromatic Elemental Imaging (Parallel Pilot).

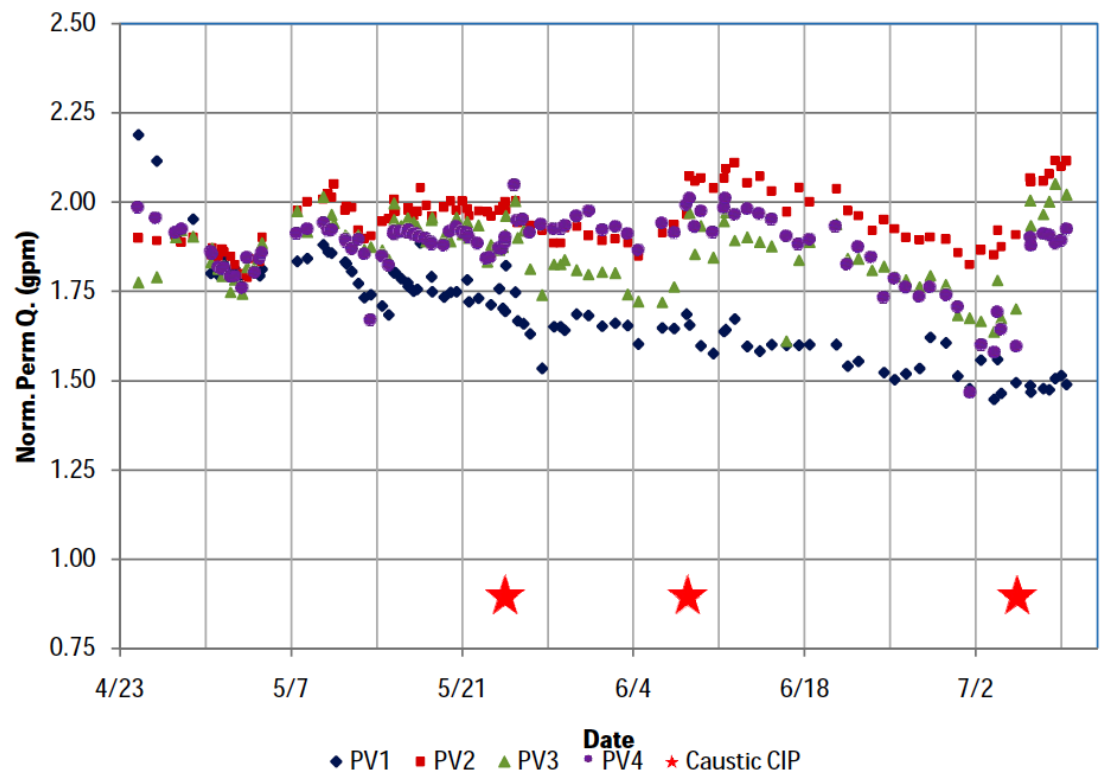

Figure 9: Parallel ROMS Pilot Performance (1 gpm=227.1 L/h).

passage was similar among the four RO membranes at approximately 1 to $2 \%$. Unlike the frequent replacement of cartridge filters in the Polishing ROMS Pilot, the head loss across cartridge filters for the Parallel ROMS Pilot was always below 0.69 bar during the total 11 week period.

\section{SUMMARY}

\section{Comparison of Pilot Scenarios}

One of major objectives of this Pilot Study was to determine the appropriate improvement alternative for the City Fargo WTP Improvement Project. A summary of the comparison between the Polishing and Parallel Scenarios has been provided in Table 3. The RO membranes performed better in the Parallel Scenario than the Polishing Scenario with respect to membrane fouling, CIP interval, flux, and recovery. It is anticipated that the Parallel Scenario will have much lower chemical and O\&M costs than the Polishing Scenario and will not have the operational challenges that were associated with the Polishing Scenario. The Parallel Scenario, therefore, has been recognized as the preferred treatment scenario. The RO design flux will 
Table 3: Comparisons of the Polishing and Parallel Pilot Scenarios

\begin{tabular}{|c|c|c|}
\hline Parameter & Polishing Scenario & Parallel Scenario \\
\hline \hline Flux Rate & $<11$ gfd not even viable & 20 to $22 \mathrm{~L} / \mathrm{m}^{2} \mathrm{~h}$ \\
\hline Recovery & $<75 \%$ & $80-82 \%$ \\
\hline Caustic CIP & 4 to 6 weeks & 10 to 12 weeks \\
\hline Acid CIP & 12 to 16 weeks & 12 to 16 weeks \\
\hline Cartridge Filter Replacement & $\sim 3$ weeks & 3 to 6 months \\
\hline Anticipated RO Membrane Life & $\sim 5$ years or less & $\sim 7$ years \\
\hline
\end{tabular}

be $12-13 \mathrm{gfd}$ and $\mathrm{RO}$ recovery will be $80-82 \%$ with consideration of the challenging water quality in the Sheyenne River. It is anticipated that the CIP interval will be 10-16 weeks and the cartridge filter replacement will be every three to six months.

\section{MF/UF Selection}

The Pilot Study verified that the MF/UF system is an essential pretreatment to improve the performance of the RO system. It was proved that the MF/UF recovery can be $95 \%$ or higher with the submerged MF/UF system that was piloted. At the instantaneous flux of 25 to $30 \mathrm{gfd}$, the CIP interval can be maintained at every 5 to 6 weeks.

\section{RO Membrane Selection}

Six different $\mathrm{RO}$ membrane modules from four well recognized RO membrane manufacturers were tested and great information was obtained for the full-scale project as well as any other future projects with similar situations. One underperformer has been identified and will be screened out for the full-scale implementation. Since the other three RO membranes performed similarly, the selection of RO membrane for the fullscale system will likely be based on a 20-year life-cycle cost analysis, which will include RO membrane installation/replacement costs, chemical costs, energy costs, and O\&M costs.

\section{REFERENCES}

[1] USGS. Simulation of the Effects of Devils Lake Outlet Alternatives on Future Lake Levels and Downstream Water Quality in the Sheyenne River and Red River of the North. Scientific Investigations Report 2011- 5050. 2011.

[2] USEPA. National Primary and Secondary Drinking Water Regulations (EPA 816-F-09-004). Washington, DC: Government Printing Office. 2009

[3] AE2S and B\&V. Fargo Water Treatment Plant Facility Plan Phase 1. 2012

[4] AE2S, Malcolm Pirnie, and University of North Dakota. Grand Forks Water Treatment Plant Ozone and BAC Filtration Pilot Study Draft Report. 2012

[5] Thunyalux Ratpukdi. Removal of Natural Organic Matter (NOM) by Ozone and Ultraviolet based Advanced Oxidation Processes: Development of NOM Fractionation Technique, Process Performances, and Bromated Formation, Ph.D. Dissertation. North Dakota State University. 2009.

[6] Craig Bartles, and Rich Franks. Understanding RO Membrane Fouling at Wastewater Treatment Plants. Proceedings of the AWWA/AMTA Membrane Technology Conference, Glendale, AZ, USA. 2012. 